\title{
Mosaicismo pigmentario del tipo hipomelanosis de Ito con hemimegalencefalia
}

\section{Pigmentary mosaicism type hypomelanosis of Ito with hemimegalencephaly}

Gabriela Zamudio-Martínez, Adriana Zamudio-Martínez, Pamela Hernández-Arriaga, Víctor Basilio LunaMacías, ${ }^{2}$ Andrea Rizo-Jiménez

Resumen

ANTECEDENTES: El mosaicismo pigmentario, del tipo hipomelanosis de Ito, es un síndrome neurocutáneo de baja prevalencia mundial caracterizado por lesiones hipocrómicas en dos o más segmentos corporales, asociados con malformaciones del sistema nervioso central, musculoesquelético, entre otros órganos y sistemas. Comparado con el resto de los síndromes neurocutáneos con patrones de herencia bien establecidos, el mosaicismo pigmentario no sigue ningún tipo de herencia, solo la manifestación esporádica es la más común. Esta enfermedad afecta a 1 de cada 7500 recién nacidos vivos, con ligero predominio por el género femenino.

CASO CLínICO: Lactante masculino de 4 meses de edad con manifestaciones cutáneas y epilepsia de difícil control, además de hallazgos por imagen, en el sistema nervioso central, de hemimegalencefalia izquierda y complejos de agiria-paquigiria sugerentes de síndrome neurocutáneo, posteriormente confirmado como mosaicismo pigmentario tipo Ito, según los criterios de Ruiz-Maldonado, mediante el reporte histopatológico de la biopsia cutánea.

PALABRAS CLAVE: Hipomelanosis de Ito; síndrome neurocutáneo; hemimegalencefalia.

\section{Abstract}

BACKGROUND: The pigmentary mosaicism of the hypomelanosis of Ito type is a low prevalence neurocutaneous syndrome characterized by hypochromic lesions in the skin, affecting two or more corporal segments, in association with malformations in the central nervous system, musculoskeletal system, as well as other organs and systems. In contrast with the other neurocutaneous syndromes with well described inheritance patterns, the pigmentary mosaicism Ito type does not have any type of inheritance, being its most common presentation the sporadic one. This disease affects 1 out of 7,500 newborns, with a mild predominance in females.

CLINICAL CASE: We present the case of a 4 month old male patient who, since birth, was noted to have hypochromic skin lesions in association with a drug resistant epilepsy, as well as imagenological findings such as hemimegalencephaly and agiria-paquigiria complexes, evoquing the possible diagnosis of a neurocutaneous syndrome, which was later confirmed as a pigmentary mosaicism or the hypomelanosis of Ito type, using the diagnostic criteria of Ruiz Maldonado, complemented with the histopathological findings in the cutaneous biopsy.

KEYWORDS: Ito hypomelanosis; Neurocutaneous syndrome; Hemimegalencephaly.
${ }^{1}$ IInstituto Tecnológico y de Estudios Superiores de Monterrey, Campus Guadalajara.

${ }^{2}$ Servicio de Pediatría, Hospital General Regional 46 (IMSS), Guadalajara, Jalisco.

Recibido: 11 de marzo 2019

Aceptado: 10 de junio 2019

Correspondencia

Gabriela Zamudio Martínez zamudio.gabriela@hotmail.com

Este artículo debe citarse como Zamudio-Martínez G, Zamudio-Martínez $A$, Hernández-Arriaga $P$, LunaMacías VB, Rizo-Jiménez A. Mosaicismo pigmentario del tipo hipomelanosis de Ito con hemimegalencefalia. Acta Pediatr Mex. 2109;40(4):217-222. 


\section{ANTECEDENTES}

El mosaicismo pigmentario del tipo hipomelanosis de Ito es un síndrome neurocutáneo de baja prevalencia que afecta a 1 de cada 7,500 recién nacidos vivos; representa el tercer síndrome neurocutáneo más común en todo el mundo, después de la neurofibromatosis tipo I y la esclerosis tuberosa. ${ }^{1}$

Este síndrome se distingue por manchas hipocrómicas que siguen las líneas de Blaschko de la piel en más de dos segmentos corporales, sin afectación de las palmas y plantas, además de malformaciones en diversos órganos y sistemas, principalmente el nervioso central, asociado con epilepsia de difícil control, discapacidad intelectual y autismo, incluso malformaciones oculares, y el sistema musculoesquelético y

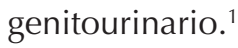

\section{CASO CLÍNICO}

Lactante de cuatro meses, nacido del segundo embarazo, sin antecedentes patológicos maternos ni antenatales de importancia; madre con embarazo de evolución normal y adecuado control prenatal. Desde el nacimiento tuvo manchas hipocrómicas en la cara y la región anterior izquierda del cuello, siguiendo las líneas cutáneas de Blaschko, sin alteraciones adicionales en otros órganos y sistemas.

Previo al alta hospitalaria, dos días después del nacimiento, comenzó con crisis focales motoras de tipo clónico en el miembro superior derecho, a razón de 50-60 por día, con duración aproximada de 1 a 2 minutos, que evolucionaron hasta afectar todo el hemicuerpo derecho, para finalmente expresar un estado parcial continuo, que no cedió con tratamiento farmacológico, por lo que se clasificó como epilepsia de difícil control, en tratamiento con midazolam, levetiracetam y oxcarbazepina sin reacción favorable.
A la exploración física se encontró una mancha hipocrómica de aproximadamente $6 \mathrm{~cm}$ en la región cervical anterior izquierda (Figura 1) y otra en la zona media-facial, en la frente, y hemiparesia espástica derecha, sugerentes de lesión focal del sistema nervioso central.

Para establecer el abordaje diagnóstico y por sospecha de síndrome neurocutáneo, por la asociación de lesiones cutáneas y manifestaciones neurológicas, se realizaron los estudios pertinentes. El electroencefalograma reportó asimetría interhemisférica por ritmo y voltaje a expensas del hemisferio izquierdo, además de paroxismos frecuentes en la región frontoparietal izquierda, sugerentes de alguna alteración estructural focal (Figura 2). La resonancia magnética mostró hemimegalencefalia izquierda y un complejo agiria-paquigiria en la región frontotemporal izquierda, compatible con los hallazgos descritos en el electroencefalograma. Figura 3

Se estableció el diagnóstico de mosaicismo pigmentario tipo hipomelanosis de Ito, con base en los criterios clínicos de Ruiz-Maldonado y sus colaboradores, ${ }^{2}$ por cumplir el criterio sine qua non de afección cutánea, y un criterio mayor de malformación en el sistema nervioso central.

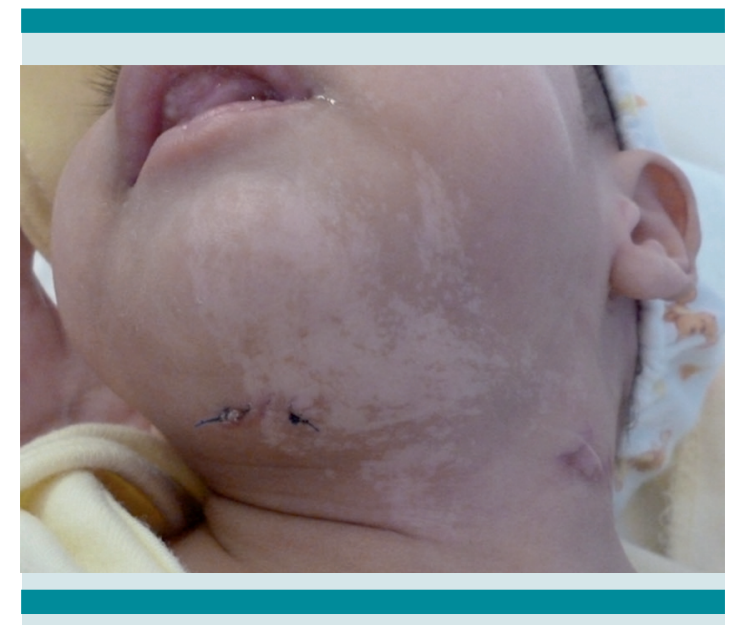

Figura 1. Mancha hipocrómica en la región cervical anterior que sigue las líneas de la piel. 


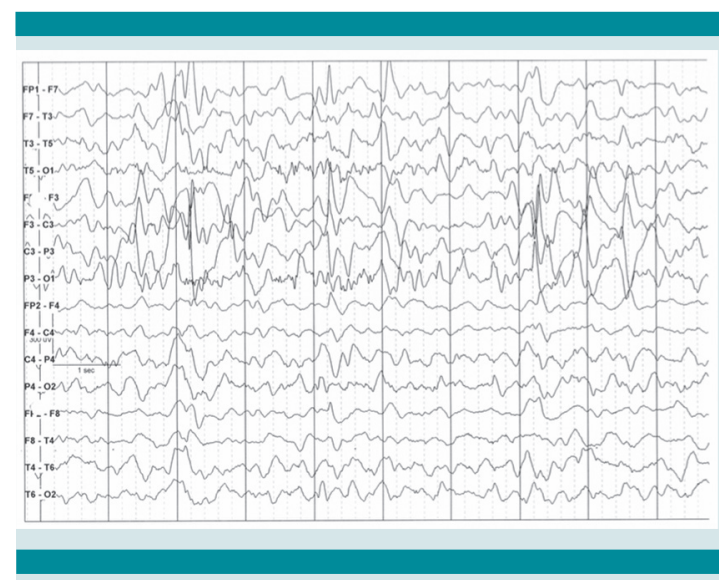

Figura 2. Electroencefalograma con asimetría interhemisférica por ritmo y voltaje, y paroxismos frecuentes en la región frontoparietal izquierda.

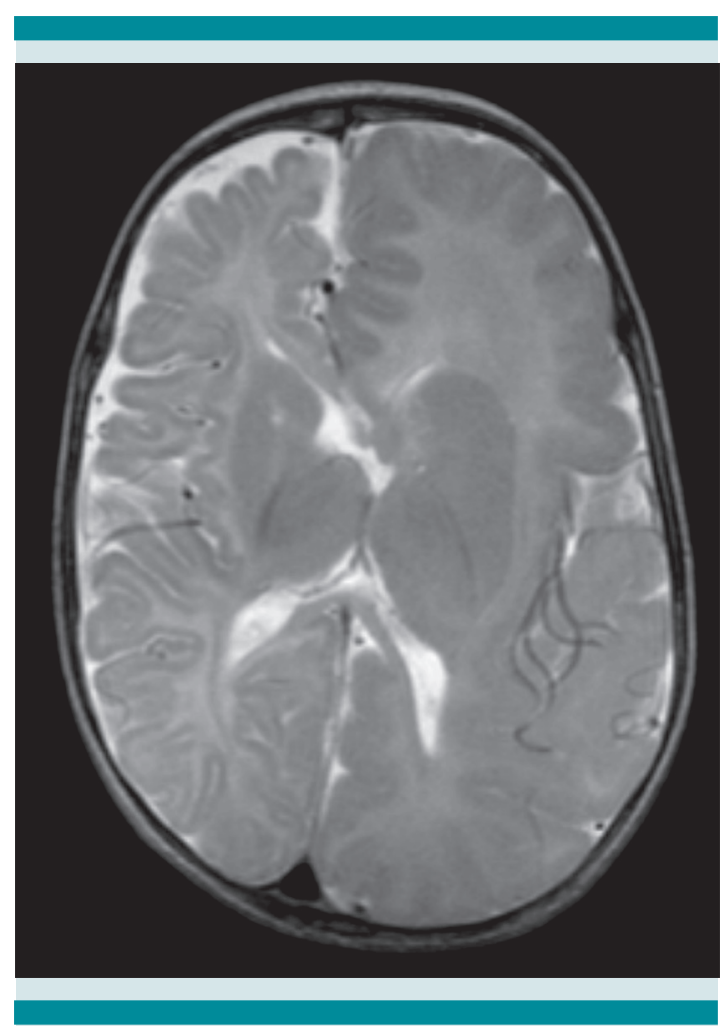

Figura 3. Resonancia magnética en secuencia T2, corte axial que muestra aumento de tamaño del ventrículo izquierdo (sobrepasa la línea media) y paquigiria en la región frontoparietal
Para complementar el diagnóstico se obtuvo una biopsia cutánea, que reportó áreas con disminución de la concentración de melanocitos y pigmento melánico adyacente a las áreas con distribución de melanocitos y pigmento normal, sin infiltrado inflamatorio, hallazgos frecuentemente encontrados en pacientes con mosaicismo pigmentario tipo Ito. No se realizaron estudios genéticos.

Debido al estado parcial continuo de resistencia a los fármacos, se decidió efectuar hemisferectomía izquierda como tratamiento definitivo de la epilepsia; sin embargo, el paciente falleció durante la intervención quirúrgica.

\section{DISCUSIÓN}

El mosaicismo pigmentario tipo hipomelanosis de Ito, también Ilamada incontinencia pigmentaria acrómica, es un síndrome neurocutáneo de baja prevalencia, descrito por primera vez en 1952, actualmente con un centenar de casos descritos en la bibliografía. ${ }^{1,3}$ Afecta a 1 de cada 7500 recién nacidos vivos, con ligero predominio por el género femenino (razón de 2:1), que lo convierte en el tercer síndrome neurocutáneo más comúnmente reportado, después de la neurofibromatosis tipo 1 y la esclerosis tuberosa. ${ }^{1}$ En este estudio se describió el síndrome en un paciente masculino, quienes reportan menor prevalencia de la enfermedad.

Este síndrome fue descrito, inicialmente, como una alteración cutánea, y posteriormente se identificó su asociación con otras malformaciones, sobre todo del sistema nervioso central y musculoesquelético; no obstante, puede afectar otros órganos y sistemas en menor medida.

Al comparar con el resto de los síndromes neurocutáneos, que tienen un patrón de herencia definido, en el mosaicismo pigmentario de tipo Ito no se ha encontrado ninguna relación genética exacta. Se han informado diferentes tipos de 
herencia, pero lo más común es la manifestación esporádica. Este síndrome se diferencia del resto de los síndromes neurocutáneos por ser un mosaicismo ectodérmico de origen monogénico o cromosómico. Sin embargo, en algunos casos se ha reportado la asociación familiar, principalmente con los genes 9q33, 15q11 y Xp11. En el proyecto "genoma humano" se reportó como una de sus principales alteraciones genéticas la translocación balanceada del cromosoma Xp21.2. ${ }^{1}$

La manifestación clínica más común es la afección cutánea, que se caracteriza por manchas hipocrómicas que afectan a más de dos segmentos corporales, siguiendo las líneas de Blaschko en los trayectos lineales o espirales, sin afectar las palmas, plantas ni mucosas. Estos hallazgos se encuentran en $100 \%$ de los pacientes afectados, en $50 \%$ desde el nacimiento y en el resto con evolución en los primeros meses de vida, motivo por el que el diagnóstico se establece de forma tardía en algunos casos. Incluso, en 30\% se han identificado manchas café con leche, ictiosis e hirsutismo. ${ }^{4,5}$ En el paciente de este estudio, las manifestaciones cutáneas aparecieron desde el nacimiento e hicieron sospechar tempranamente de algún síndrome neurocutáneo, por su asociación con crisis convulsivas.

Las manifestaciones extracutáneas más frecuentes son las que afectan el sistema nervioso central (94\%), sobre todo epilepsia focal de difícil control, secundaria a malformaciones corticales como: paquigiria y agiria, agenesia del cuerpo calloso y hemimegalencefalia asociada o no con hemihipertrofia corporal. Estas alteraciones estructurales pueden identificarse mediante electroencefalograma en $50 \%$ de los casos. ${ }^{6}$ Nuestro paciente tuvo malformaciones cerebrales tipo agiria-paquigiria y hemimegalencefalia izquierda, que condicionaron la epilepsia resistente al tratamiento desde los primeros días de vida.
Desde el punto de vista neuropsiquiátrico estas malformaciones cerebrales pueden manifestarse con discapacidad intelectual, trastornos del comportamiento y autismo. ${ }^{3}$ Se ha documentado que $60 \%$ de los pacientes afectados tiene un coeficiente intelectual menor de 70 y el otro $15 \%$ tiene inteligencia limítrofe. ${ }^{7}$ En una serie de 76 casos, Castroviejo y sus coautores reportaron que sólo $22 \%$ de los pacientes tenía inteligencia en límites de normalidad. ${ }^{8}$

El sistema musculoesquelético representa el segundo sistema extracutáneo más afectado, con alteraciones en la columna vertebral (cifosis o escoliosis) y el esqueleto axial (sindactilia y polidactilia). Una manifestación rara es la hipertrofia hemicorporal.

Por tratarse de una enfermedad sistémica pueden encontrarse alteraciones que afectan cualquier parte del organismo; en la bibliografía se han descrito hallazgos dentales, genitourinarios y cardíacos, no encontrados en nuestro paciente. ${ }^{9}$

En la mayoría de los casos el diagnóstico se establece clínicamente, con los criterios de Ruiz-Maldonado, ${ }^{2}$ donde el criterio sine qua non se distingue por manchas hipocrómicas en dos o más segmentos corporales, y como parte de los criterios diagnósticos menores, cualquier malformación anatómica en otros órganos y sistemas diferentes al nervioso central y musculoesquelético. ${ }^{2}$ (Cuadro 1) En algunos casos la biopsia cutánea puede ser útil para complementar el diagnóstico, al encontrar zonas de piel hipopigmentada, con disminución o ausencia total del pigmento cutáneo secundario a la baja concentración de melanocitos, sin infiltrado inflamatorio. ${ }^{10}$ En el paciente de este estudio se estableció el diagnóstico temprano, en los primeros meses de vida, al identificar el criterio sine qua non de la enfermedad, así como malformaciones en el sistema nervioso central, que posteriormente se sustentó con los hallazgos en 
Cuadro 1. Criterios diagnósticos de mosaicismo pigmentario, tipo hipomelanosis de Ito, propuestos por Ruiz-Maldonado en $1992 .{ }^{9}$

\begin{tabular}{l|l} 
Criterios & \multicolumn{1}{c}{ Hallazgos } \\
\hline Criterio sine qua non & $\begin{array}{l}\text { Hipopigmentación congénita o adquirida precozmente, no hereditaria, que afecta } \\
\text { dos o más segmentos corporales. }\end{array}$ \\
\hline Criterios mayores & $\begin{array}{l}\text { Una o más alteraciones en el sistema nervioso central. } \\
\text { Una o más alteraciones musculoesqueléticas. }\end{array}$ \\
\hline Criterios menores & $\begin{array}{l}\text { Dos o más manifestaciones congénitas diferentes en el sistema nervioso central y } \\
\text { musculoesquelético }\end{array}$ \\
\hline Diagnóstico definitivo & $\begin{array}{l}\text { Criterio sine qua non más uno o más criterios mayores. } \\
\text { Criterio sine qua non más dos o más criterios menores. }\end{array}$ \\
\hline Diagnóstico presuntivo & Criterio sine qua non asociado con un criterio menor
\end{tabular}

la biopsia cutánea. Sólo 50\% de los pacientes manifiesta las manchas hipopigmentadas características desde el nacimiento. ${ }^{4}$

La hemimegalencefalia es una malformación del sistema nervioso central caracterizada por el crecimiento anormal de un hemisferio cerebral, que puede aparecer de manera aislada o relacionado con el mosaicismo pigmentario tipo Ito. Esta malformación provoca manifestaciones neurológicas: epilepsia de difícil control, hemiparesia y discapacidad intelectual. La epilepsia relacionada con hemimegalencefalia suele ser resistente al tratamiento y los pacientes pueden requerir hemiesferectomía, cuyo pronóstico es sombrío, a pesar del tratamiento quirúrgico. El inicio temprano de la epilepsia y la duración de las crisis convulsivas son factores de mal pronóstico asociados con inadecuado control después de la cirugía. ${ }^{11}$ Nuestro paciente tenía ambos factores, con epilepsia de inicio en el segundo día de vida y estado parcial continuo de resistencia a los fármacos.

Hasta el momento no existe ningún tratamiento para el mosaicismo pigmentario tipo lto; sin embargo, el diagnóstico oportuno y la intervención de un equipo multidisciplinario puede asociarse con mejor pronóstico y menor morbilidad. ${ }^{12}$ En el caso aquí expuesto, a pesar del diagnóstico temprano (primeros meses de vida), las malformaciones cerebrales condicionaron la elevada morbilidad, con el estado epiléptico parcial continuo que requirió tratamiento quirúrgico, y un desenlace mortal.

\section{CONCLUSIONES}

Los síndromes neurocutáneos son alteraciones de baja prevalencia, con manifestaciones clínicas cutáneas y del sistema nervioso central, que pueden condicionar trastornos neurológicos: epilepsia de difícil control, discapacidad intelectual y autismo. El diagnóstico de síndrome neurocutáneo debe sospecharse en pacientes con lesiones cutáneas hipocrómicas y alteraciones del sistema nervioso central. La afectación neurológica puede manifestarse como epilepsia de difícil control o alteraciones en los estudios de imagen. En algunos tipos de síndromes neurocutáneos, como el mosaicismo pigmentario tipo lto, puede establecerse el diagnóstico únicamente con los hallazgos clínicos. Hasta la fecha no existe un tratamiento específico; sin embargo, la importancia del diagnóstico temprano radica en el inicio oportuno del tratamiento y de las comorbilidades que puedan aparecer. 


\section{REFERENCIAS}

1. Romero A, et al. Hipomelanosis de Ito. Dermatol Rev Mex 2015; 59:43-48. https://nietoeditores.com.mx/nieto/Dermatologia/2015/ene-feb/caso.clinico_hipomelanosis.pdf

2. Ruiz-Maldonado $R$, et al. Hypomelanosis of ito: diagnostic criteria and report of 41 cases. Pedatr Dermatol 1992;9(1):1-10.

3. Díaz-Victoria $A R$, et al. Análisis neuropsicológico de la hipomelanosis de Ito. Estudio de caso. Rev Mex Neuroci 2007;8(1):86-90.

4. Kumar HY, et al. Hypomelanosis of Ito: A rare cutaneous syndrome. Int J Health Allied Sci 2013;2:203-5. DOI: $10.4103 / 2278-344 X .120591$

5. López-Blanco MA, et al. Hipomelanosis de Ito, reporte de un caso. Derm Venez 1996;34:121-125.

6. Steiner J, et al. Hipomelanosis of Ito and brain abnormalities: MRI findings and literature review. Pediatr Radiol 1996;26:763-768.
7. Llamos Paneque A, et al. Hipomelanosis de Ito. MEDISAN 2002;6(4):82-85.

8. Pascual-Castroviejo I, et al. Hypomelanosis of Ito. A study of 76 infantile cases. Brain Dev Jpj 1998;20(1):36-43.

9. Ruggieri M, Pavone L. Hypomelanosis of Ito: clinical syndrome or just phenotype?. J Child Neurol. 2000;15(10)635-644.

10. Donnai $D$, et al. Hypomelanosis of Ito: a manifestation of mosaicism or chimerism. J Med Genet1988;25:809-818

11. Ikeda KM, et al. Evolution of epilepsy in hemimegalencephaly from infancy to adulthood: Case report and review of the literature. Epilepsy Behav Case Rep 2017;7:4548. https://doi.org/10.1016/j.ebcr.2017.02.002

12. Koehler $\mathbf{M}$, et al. Hypomelanosis of ito in two infants: a case series with literature review. J Am Osteopath Col Derm 2015;32:49-51. https://cdn.ymaws.com/ www.aocd.org/resource/resmgr/jaocd/contents/volume32/32-14.pdf 